\title{
Obesidade osteosarcopênica sob o olhar da Nutrição Funcional
}

- Por muito tempo, os estudos científicos analisavam características ósseas, musculares e adiposas de forma separada. Porém atualmente reconhece-se uma ligação entre esses três pontos, na qual um pode interferir na funcionalidade dos demais;

- Em um indivíduo saudável existe um equilíbrio no funcionamento dos osteoblastos, miócitos e adipócitos, assim como um equilíbrio na distribuição e infiltração de gordura nesses tecidos;

- Com o envelhecimento, observa-se um maior acúmulo de gordura no tecido adiposo, e uma menor atividade de osteoblastos e miócitos. No ponto de interseção entre os 3 sistemas inicia-se uma inflamação de baixo grau que, uma vez presente de forma crônica, pode comprometer os três tecidos e suas funções;

- Com a progressão desse quadro inflamatório, surge o que os autores definem como obesidade osteosarcopênica, que seria o ponto central de interseção entre problemas ósseos, musculares e obesidade;

- A obesidade osteosarcopênica pode trazer alguns impactos sérios para o paciente, como aumentar o risco de quedas e fraturas, fragilidade, diminuição da capacidade funcional e física, aumento de morbidade, mortalidade, redução da qualidade de vida, além de desordens metabólicas, como resistência à insulina, síndrome metabólica, dislipidemia, hipertensão arterial, entre outras;

- Essa condição também está associada com redução de mobilidade, redução do gasto energético, perda de massa magra, inflamação, resistência à insulina e acúmulo de gordura, e, pensando nesses aspectos, tanto exercício quanto nutrição representam importantes alvos terapêuticos;

- Não existe uma distribuição ideal de macronutrientes, mas estudos destacam a importância de dar atenção para o consumo proteico, além da ingestão adequada de fibras e ômega-3;

- Uma ingestão inadequada crônica de micronutrientes pode afetar músculo, osso e gordura e causar ou agravar um quadro de obesidade osteosarcopênica. Os principais micronutrientes citados são: cálcio, magnésio, ferro, fósforo, vitaminas B6, B12, C e as lipossolúveis A, D, E e K.

\section{Referências bibliográficas}

1. JAFARINASABIAN, P.; INGLIS, J.E.; KELLY, O.J.; ILICH, J.Z. Osteosarcopenic obesity in women: impact, prevalence, and management challenges. Int J Womens Health; 9: 33-42, 2017.

2. ILICH, J.Z.; KELLY, O.J.; INGLIS, J.E. Osteosarcopenic Obesity Syndrome: What Is It and How Can It Be Identified and Diagnosed? Current Gerontology and Geriatrics Research; 2016: 7325973, 2016.

3. POLYSOS, S.A.; MARGIORIS, A.N. Sarcopenic obesity. Hormones (Athens); 17(3): 321-331, 2018.

4. ORMSBEE, M.J.; PRADO, C.M.; ILICH, J.Z. et al. Osteosarcopenic obesity: the role of bone, muscle, and fat on health. J Cachexia Sarcopenia Muscle; 5(3): 183-192, 2014.

5. TROUWBORST, I.; VERREIJEN, A.; MEMELINK, R. et al. Exercise and Nutrition Strategies to Counteract Sarcopenic Obesity. Nutrients; 10(5): 605, 2018.

6. KELLY, O.J.; GILMAN, J.C.; KIM, Y.; ILICH, J.Z. Macronutrient Intake and Distribution in the Etiology, Prevention and Treatment of Osteosarcopenic Obesity. Curr Aging Sci; 10(2): 83-105, 2017.

7. KELLY, O.J.; GILMAN, J.C.; KIM, Y.; ILICH, J.Z. Micronutrient Intake in the Etiology, Prevention and Treatment of Osteosarcopenic Obesity. Curr Aging Sci; 9(4): 260-278, 2016. 